\title{
Research on Mechanism of Detecting Communication Network of Power Remote Automatic Meter Reading
}

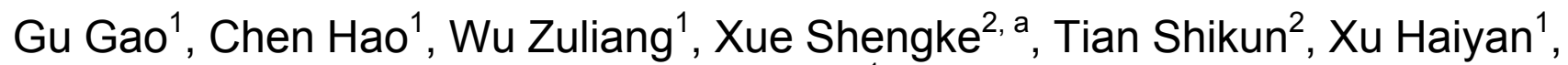 \\ Jin Yulan ${ }^{1}$ \\ ${ }^{1}$ State Grid Corporation of China in Shaoxing, Shaoxing, 312000, China \\ ${ }^{2}$ College of Information Science and Electronic Engineering, Zhejiang University, Hangzhou, \\ 310027, China \\ a email: xueshengke@zju.edu.cn,
}

Keywords: power line carrier, GPRS, wireless network, routing algorithms, parameters detecting

\begin{abstract}
With the launch and implementation of the intelligent grid, the remote meter reading through power line carrier and GPRS wireless network has been widely deployed gradually. However, the wireless network based on SIM card is vulnerable to interference at present, the packet loss and drop occurring sometimes, and due to the complex environment and enormous attenuation of the grid network, the requirements of high quality communication are not guaranteed. Therefore, it is one of the significant technologies that the effective method of detecting communication network of the power remote automatic meter reading. Analyses the features and parameters of GPRS wireless network for the power information collection simultaneously, researches mainly on the comprehensive detecting communication network of the power remote automatic meter reading and power line carrier routing. Evaluate status of the communication of the remote meter reading supported by the statistics from power line carrier network, including the time of routing, communication success rate and communication delay.
\end{abstract}

\section{Introduction}

The power information collection and management system is the one that collecting and monitoring the power information from multiple meters through transmission carrier by master station. It consists of the power meter, terminal, concentrator, master station and so on, which is the system with the functions including power automatic data acquisition, measurement anomalies, power quality monitoring, analysis and management of electricity [1].

The power information collection and management system is composed of three layers of physical structure shown in Figure 1.

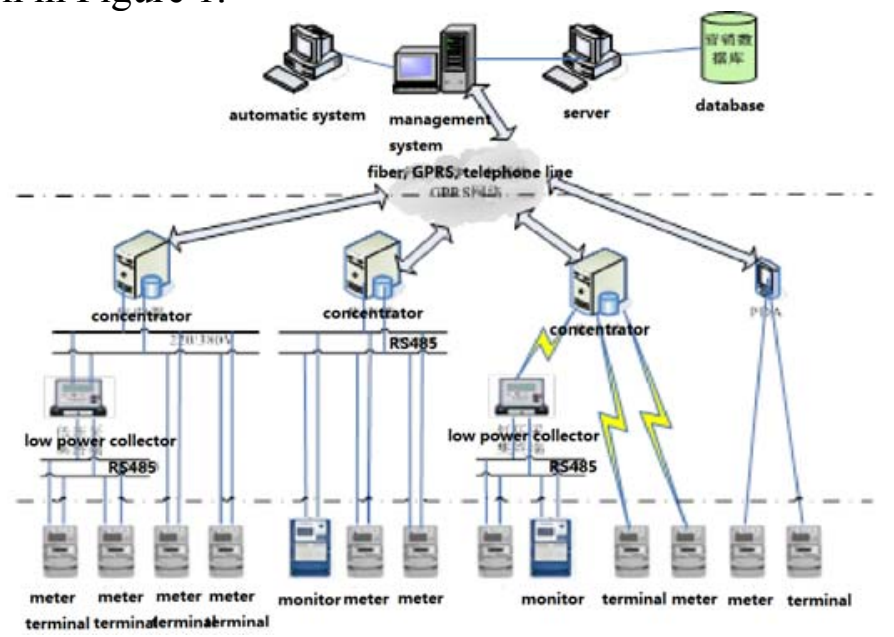

Fig.1. The physical structure of power information collection and management system

The first layer, master station, administrative center of the entire system, is responsible for the power information collection, power management, statistics management and applications. The 
second layer, data acquisition layer, in charge of power information collection and monitoring from the collection points, including a variety of applications of power information collection terminals. The third layer, is the information sources and monitoring objects [2] of the power information in collection points, such as meters, associated measurement equipment, power distribution switches, reactive compensation devices and other intelligent field devices. The communication network accomplishes the data transmission between layers, which may be the private or public wireless, wired communication network and power line carrier communication network.

\section{Communication Network of the Power Remote Meter Reading}

GSM is the largest and the most extensive network in the world. The data transmission of GSM relies on the circuit-switched technology [3], while the packet switching technology for GPRS, which is able to track the location of a mobile station, perform security, access control, authentication of the mobile station and mobile management, route, transmit the information from GPRS network to GPRS terminal, connect to the Internet network by driving GPRS module, and implement the power information transmission via Internet eventually [4]. The data is wrapped into IP packets at first if the meter reading device sends data to server, and encapsulates IP datagram to PPP frame through PPP protocol then. After sent to GPRS wireless module by serial port, PPP frames will be processed for IP broadcast. Initialization, network access and data transmission of the GPRS wireless module are done via AT commands, which are extensively covered, suitable for moving objects, easy to install and maintain, and has been widely used in lots of applications [5].

Since disturbed by Doppler effect, fading, jitter and noise of electromagnetic field, there is a certain probability of error and dropping in the radio channel. This paper adopts an original mechanism of detecting multiple channels of remotely collecting power information simultaneously, which measures and judges synthetically by adopting the method that verifying data, retransmitting more than once in a cycle time and comparing with previous data received, and reinforces the performance of detecting the real-time status of wireless network communication. By the control and judgment of GPRS wireless module processes, the method detects status of the transportation, in particular A) the control module initializes GPRS wireless module through AT commands, so that it can attach the GPRS network, obtain IP address of the GPRS terminal from dynamically allocation by network operators, request the connection to server in management center, commence two-way data transmission in real-time after establishing the connection with management center; B) the control module sends instructions or receives data from underlying network via M-Bus interface module; C) the control module exchanges data to the TCP / IP module; D) the control module meets user's needs of different kinds of meter reading by RS232 module, meter reading and setting aside the interface; E) before accessing the Internet, the control module is configured certainly adopting AT + I commands first, and the system records all of access, process operations, including user ID, IP, time, digital signatures, operating items and other information. Constructing the monitoring and control of multiple channels simultaneously, the overall communication channel status of GPRS wireless network can be obtained.

Detecting the parameters related to communication processes of mobile routing in power information transmitted in GPRS network, which bases on the construction and implementation of mobile communication core network system. But the remote meter reading of power information is accomplished by power line carrier communication primarily, towards the areas that lacking coverage of the GSM mobile network or with poor coverage quality. Power line carrier communication is a cable transmission network with wide distribution, multiple users and high variability, but the low-voltage power lines are not designed to transmit data. Its topology and physical characteristics are different from the traditional transmission media such as twisted pair, coaxial cable, fiber, etc. When transmitting signals, the channel characteristics are quite complicated, have multi-load, strong noise and interference, attenuation, channel delay, so that the requirements of carrier communication are hard to meet in many ways [6]. Although the power information collection system based on power line carrier communication is able to improve the communication quality to some extent via adopting efficient anti-interference spread spectrum modulation 
techniques, in practical application, it conforms with the low power environment of our country that building a suitable mechanism in network layer applications in order to increase the reliability of communication further. The key technology is to optimize and configure the route of power line carrier communication based on the measurement to status of power carrier. At the moment, the routing algorithms applied in power line carrier communication are sorted into two categories, one for hierarchical routing algorithms, such as flooding algorithm [7] and clustering algorithm [8], another for distributed routing algorithms, like ant colony algorithm [9] [10].

(1) Flooding algorithm

Currently, the flooding algorithm is widely used in power line carrier routing protocols, which can find a path to the destination node in any case with a short delay. But there is a deficiency: the broadcast storm, which means that all nodes in the network are involved in forwarding packets i.e. broadcast, contributing to the number of network packets rises in geometric magnitude, and the situation of network deteriorates sharply because of packets spreading repeatedly like flood.

(2) Clustering algorithm

The basic idea of clustering algorithm is dividing the network into several clusters logically to solve network issues of the low power line grid. For raising the survivability and reliability of power line carrier communication, a routing algorithm with hierarchical design, cluster management and automatic relay is suggested, which adopts the non-overlapping clustering algorithm, implementing the dynamic routing of low power line carrier communication system.

(3) Ant colony algorithm

Ant colony algorithm, also known as ant colony optimization algorithm. The artificial ant in algorithm not only has the capability of searching, also has memory and the inspired computing power. Memory allows the ant remember its route, while inspired computing capability makes ant evaluate the benefit of choosing the next path to the final solution. After all ants have accomplished their search, the stage enters, updating the routing table, global releasing and volatile in accordance with the principle of concentration of pheromone. If the number of iteration is maximum or meets the requirement of system, the search terminates, otherwise re-releasing all ants and beginning the next round of search. References [11] [12] suggest a design of adaptive routing based on power line carrier networks through using ant colony thought, and get good results.

\section{Design of Detecting Parameters}

In order to adapt the environment of power line carrier communication, this paper proposes the detection of quality of network communication in the case of applying different routing algorithms or no algorithms. These parameters become the reference of evaluating, selecting and amending routing algorithms.

Determine whether it is necessary to deploy the routing algorithms for improving the quality of network communication, by detecting communication success rate and delay of each node, when none algorithm exists. But time of routing, communication success rate, and communication delay of each node are beneficial to amend the routing algorithms when it has worked in network.

This paper selects three indicators as follows:

(1) Time of routing Tc: time for searching the best path in the power line carrier network with particular routing algorithm when automatically reported from nodes in initialization. This indicator is used for assessing the speed of searching and updating of the routing algorithm, by the way, it also relates to the number of nodes in the network:

$$
T_{c}=N \bullet t_{s}
$$

$\mathrm{N}$ is the total number of nodes in the network. Nodes include power meter, collector, and other communication units deployed with routing algorithm. The search time for the node in average is $t_{s}$. The search time in average is short, indicating that the speed of search is fast and the routing process is efficient.

(2) Node communication success rate R: it is the most intuitive and significant indicator in the measurement of communication quality of a node of communication network. It defines as the ratio 
of the number of successful communication with the total number from concentrator to a node, in a detecting period. Success is demonstrated as the concentrator sends the total active power instruction, the meter correctly responds, and the concentrator receives:

$$
R=X / Y
$$

$\mathrm{X}$ is the number of successful communication of a node within a certain period, and $\mathrm{Y}$ is the total number of a node correspondingly.

(3) Communication delay in average $\mathrm{Td}$ : communication delay is another indicator in the measurement of performance of communication network, which describes the transmission time of data packets consumed from transmitter to receiver, and the smaller number it gets, the better performance it reveals. This paper defines the communication delay as the time from the concentrator sending reading instruction until receiving the correct response of meter. If fails, it records nothing. Accumulate a lot of results in a detecting period, and get the number in average ultimately:

$$
T_{d}=\left(\sum_{i=1}^{X} t_{i}\right) / X
$$

$t_{i}$ is communication delay in one time.

Definition of detecting period T: the length of detecting time is set by user, and the concentrator tests $\mathrm{Td}$ and $\mathrm{R}$ in a detection period unit. For one point, test the same power meter repeatedly, and record the total number $\mathrm{Y}$, the number of successes $\mathrm{X}$, and the cumulative communication delay. For many points, test all of power meters in network, and record their total number $Y$, success the number X, and the cumulative communication delay separately. During each detecting period T, a meter has its $\mathrm{R}$ and $\mathrm{Td}$ correspondingly. Detecting period may be 30 minutes, one hour, etc., arbitrarily set by the user.

\section{Test in various routing environment}

The power line carrier detection is aimed to provide a professional communication detection system of power line carrier route, capable of testing the quality of network and its routing algorithms, obtains the operating characteristics data, thus the quality of a power line carrier communication network can be assessed .

Test follows these cases:

(1) Whether the route exists or not, or different routing algorithms performs;

(2) Time-variant carrier network characteristics.

To this end, the following three situations are offered for testing:

(I) No route: concentrator, copy controller, power meter;

(II) The network environment based on routing algorithm A: concentrator, carrier routing master module, power meter;

(III) The network environment based on routing algorithm B: concentrator, carrier routing master module (programmed with new routing algorithms), power meter.

It should be clarified that the routing algorithm $\mathrm{A}$ is an old version, which implements the basic functions like looking to achieve a relay and relay communication. However the routing algorithm B is optimized from algorithm A, which accelerates the speed of propagation, reduces the time of routing, and chooses the optimal path in multi-hop routing.

Prepare a concentrator, 7 power meters with the carrier communication modules, coded from 01 to 07 , a copy controller, two carrier routing master modules with different routing algorithms and several attenuators; Topology is shown in Figure 2. In the figure, arrow indicates connection between two nodes so that the carrier communication is established directly. To the contrary, no arrow shows attenuator by $80 \mathrm{~dB}$ is placed between them cutting off the carrier communication. There are no other electrical equipment running in the environment. 


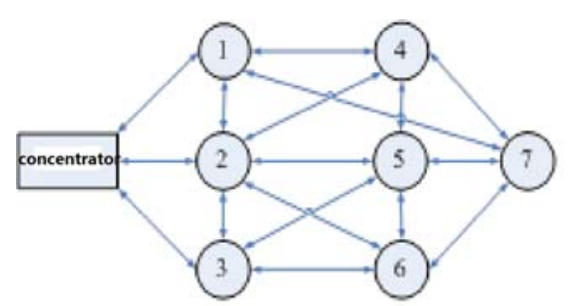

Fig.2. The topology structure

The number of relay from concentrator to meter 1 to 3 is 0 , i.e., can communicate directly. The number of relay from concentrator to meter 4 to 6 is 1 , and the communication can be realized through one hierarchy of relay. The number of relay from concentrator to meter 7 is 2 : concentrator $->$ meter 1 -> meter $4->$ meter 7 , or concentrator $->$ table $1->$ table 7 as one hierarchy of relay.

Detect on this topology under the circumstance I, II and III, execute collecting meters 1 to 7 each for 1000 times, and record communication success rate, communication delay, the results of statistics are shown in Table 1, Table 2 and Table 3:

Tab.1. result of situation I

\begin{tabular}{|c|c|c|}
\hline parameter & $\begin{array}{c}\text { communication success rate } \\
\mathrm{R} \text { in average }\end{array}$ & $\begin{array}{c}\text { communication delay } \\
\text { Td in average/second }\end{array}$ \\
\hline meter 1 & 0.924 & 1.8 \\
\hline meter 2 & 0.910 & 1.7 \\
\hline meter 3 & 0.946 & 2.1 \\
\hline meter 4 & 0 & N/A \\
\hline meter 5 & 0 & N/A \\
\hline meter 6 & 0 & N/A \\
\hline meter 7 & 0 & N/A \\
\hline
\end{tabular}

Tab.2. result of situation II

\begin{tabular}{|c|c|c|}
\hline parameter & $\begin{array}{c}\text { communication success rate } \\
\mathrm{R} \text { in average }\end{array}$ & $\begin{array}{c}\text { communication delay } \\
\mathrm{Td} \text { in average/second }\end{array}$ \\
\hline meter 1 & 0.974 & 1.8 \\
\hline meter 2 & 0.966 & 2.0 \\
\hline meter 3 & 0.958 & 2.1 \\
\hline meter 4 & 0.935 & 4.2 \\
\hline meter 5 & 0.953 & 3.9 \\
\hline meter 6 & 0.914 & 4.4 \\
\hline meter 7 & 0.876 & 5.6 \\
\hline
\end{tabular}

The time of routing in situation II: 126 seconds. 
Tab.3. result of situation III

\begin{tabular}{|c|c|c|}
\hline parameter & $\begin{array}{c}\text { communication success rate } \\
\mathrm{R} \text { in average }\end{array}$ & $\begin{array}{c}\text { communication delay } \\
\text { Td in average/second }\end{array}$ \\
\hline meter 1 & 0.986 & 1.8 \\
\hline meter 2 & 0.992 & 1.7 \\
\hline meter 3 & 0.978 & 1.7 \\
\hline meter 4 & 0.975 & 3.4 \\
\hline meter 5 & 0.981 & 3.2 \\
\hline meter 6 & 0.964 & 3.5 \\
\hline meter 7 & 0.936 & 3.8 \\
\hline
\end{tabular}

The time of routing in situation III: 85 seconds.

By analyzing the data above, it comes to a conclusion that the concentrator can merely receive a copy from the meter directly connected in the network with no route, and fails to communicate with other meters; Communication delay maintains about 1.8 seconds; The concentrator is able to collect all meters in network with routing algorithm $\mathrm{A}$, with respect to the number of relay to 0 , relay number 1 makes communication delay increase 115 percent while success rate decrease 4.2 percent; The concentrator is capable to collect all meters in network with routing algorithm $\mathrm{B}$, the communication delay ascends 187 percent but success rate descends by $9.4 \%$ when comparing relay number 2 with the number of relay to 0 . It is obvious that routing algorithm $B$ has significant improvement to A, and similarly for network with route to no route.

\section{Conclusion}

Detecting the parameters related to the communication processes of mobile routing in the power information transmitted in GPRS network, which bases on the construction and implementation of mobile communication core network system. But the remote meter reading of power information is accomplished by the power line carrier communication primarily, towards the areas that lacking the coverage of the GSM mobile network or with poor coverage quality. Power line carrier communication is a cable transmission network with wide distribution, multiple users and high variability. The detecting application of power line carrier is supposed to detect the network about the characteristics of the channel attenuation, the performances under different routing algorithms and its time variability. Acquiring the statistics about the time of routing Tc, communication success rate $\mathrm{R}$ and communication delay $\mathrm{Td}$, which is the unique part of the application, contributes to the fault diagnosis of the power line carrier communication network and the optimization of the routing algorithms.

\section{References}

[1] Tian Shikun. Research of Embedded Concentrator with PLC Detection [D]. Zhejiang University, 2011.

[2] Nicholson J, Malack J. RF impedance of power lines and line impedance stabilization networks in conducted interference measurements[J]. IEEE Transactions on Electromagnetic Compatibility, 1973, 2(EMC-15): 84-86.

[3] Duan Jin, Ye Lin, Huang Xianshu. Remote data sampling and transmission system based on GSM - short message service [J]. Journal of Changchun Institute of Optics and Fine Mechanics, 2006, 28(4): 51-53.

[4] Han DongFang, Zhu Shanan, Jiang Zhengyi. The terminal design of wireless meter reading system based on GPRS / SMS [J]. Industrial Control Computer, 2005, 17(10): 28-29.

[5] Meng Xiaoning, Wang Yongbin, Sun Jianrong. Wireless data communication and applied foreground based on GPRS[J]. Modern Electronic Technique, 2005, 28(19): 31-33.

[6] Qi Jiajin, Chen Xueping, Liu Xiaosheng. Advances of research on low-voltage power line 
carrier communication technology[J]. Power System Technology, 2010, 34(5): 161-172.

[7] Yassein M B, Ould-Khaoua M, Papanastasiou S. On the performance of probabilistic flooding in mobile ad hoc networks[C]. Parallel and Distributed Systems, 2005. Proceedings. 11th International Conference on. IEEE, 2005, 2: 125-129.

[8] Gao Li, Mu Dejun, Zhang Li, Zhang Guoqing. Novel clustering algorithm in ad-hoc network and performance evaluation[J]. Computer Science, 2009, 36(5): 82-84.

[9] Dorigo M, Maniezzo V, Colorni A. Ant system: optimization by a colony of cooperating agents[J]. Systems, Man, and Cybernetics, Part B: Cybernetics, IEEE Transactions on, 1996, 26(1): $29-41$.

[10] Dorigo M, Gambardella L M. Ant colony system: a cooperative learning approach to the traveling salesman problem[J]. Evolutionary Computation, IEEE Transactions on, 1997, 1(1): 53-66.

[11] Liu Xiaosheng, Zhou Yan, Qi Jiajin. Method study of automatic routing for power line communication[J]. Proceedings of the CSEE, 2007, 26(21): 76-81.

[12] Wang Li, Yang Chunyan, Chen yue. The researching of the low-voltage power line carrier adaptive routing algorithm owning to the $\mathrm{ACA}[\mathrm{J}]$. World Chinese Entrepreneur Economic Yearbook · Gaoxiao Jiaoyu Yanjiu, 2008 (10). 